\title{
El género Roccella en la Península Ibérica y las Islas Baleares
}

\author{
Regina Carballal ${ }^{1}$
}

Abstract: Carballal, R. 2013. The genus Roccella in the Iberian Peninsula and Balearic Islands. Bot. Complut. 37: 13-20.

\begin{abstract}
The morphological, anatomical, chemical and geographical data of 6 species of genus Roccella is reported from the Iberian Peninsula and Balearic Islands. An identification key based in macroscopic characters and chemical reactions is presented. Roccella elisabethae is recorded for the first time from continental Europe.
\end{abstract}

Key words: lichens, Roccella, Iberian Peninsula, Balearic Islands.

Resumen: Carballal, R. 2013. El género Roccella en la Península Ibérica y las Islas Baleares. Bot. Complut. 37: 13-20.

Se aportan datos morfológicos, anatómicos, químicos y de distribución de 6 especies del género Roccella de la Península Ibérica e islas Baleares. Se presenta una clave de identificación basada en caracteres macroscópicos y reacciones químicas. Roccella elisabethae se cita por primera vez para el continente europeo.

Palabras clave: líquenes, Roccella, Península Ibérica, Islas Baleares.

\section{INTRODUCCIÓN}

Roccella DC. es el género tipo de la familia Roccellaceae (Arthoniales). Comprende unas 20 especies (Tehler \& Irested 2007), todas ellas de ambientes costeros con distribución subtropical, pero más frecuentes en las áreas de clima mediterráneo del Hemisferio Norte. El género, homogéneo y bien definido, se caracteriza por su talo fruticuloso de ramas planas o cilíndricas, fijado al sustrato por un único disco basal, cuya médula (hipomédula) presenta coloraciones variables según la especie. El fotobionte es siempre Trentepohlia. Los ascomas son apotecios, con o sin excípulo talino, frecuentemente pruinosos, con un hipotecio muy desarrollado, castaño o negro y un epitecio también negro. Los ascos, fisitunicados, contienen 8 ascósporas, fusiformes, a veces curvadas, triseptadas. En algunas especies se forman picnidios con conidios filiformes. Son muy comunes los soralios, algunas veces en los mismos individuos que presentan apotecios, aunque lo más frecuente es que la reproducción vegetativa y la sexual se encuentren en talos diferentes. Esto motivó la consideración de «especies par» actualmente en su mayoría unificadas en un único taxón.

Algunos caracteres utilizados para diferenciar especies en las claves del género para Europa y Macaronesia son difíciles de observar, lo que unido a errores de denominación lleva a confusiones en la identificación. Esto es particularmente notorio en los referidos a la anatomía del córtex; en Roccella se presentan dos tipos de córtex: en algunas especies se forma un plecténquima en empalizada con todas las hifas iguales, dispuestas perpendiculares a la superficie, de aspecto muy homogéneo, en otras, las hifas se entrelazan, algunas son transversales y su aspecto no es regular como en el caso anterior. Según Tavares (1958) estos caracteres se aprecian bien en los extremos de las ramas y su observación es difícil en las partes medias y basales de las mismas. Una opinión parecida mantienen Aptroot \& Schumm (2011), que recomiendan realizar tinciones y secciones, tanto transversales como longitudinales. Además, un error en la denominación de los dos tipos de córtex: «tipo tinctoria», el primero y «tipo canariensis» el segundo (Follmann 1969), nombres tomados del par de especies $R$. tinctoria/R. canariensis que recientemente han sido unificadas (Tehler et al., 2009a), complica todavía más la identificación.

En algunas especies se forma un epicórtex gelatinoso, claramente visible en las secciones transversales del talo, aunque más tenue en los ápices de las ramas; por debajo se encuentra el córtex celular, denominado subcórtex, que suele ser del tipo plecténquima de hifas entrela-

\footnotetext{
${ }^{1}$ Departamento de Botánica. Universidad de Santiago de Compostela. mregina.carballal@usc.es

Recibido: 13 febrero 2013. Aceptado: 5 marzo 2013.
} 
zadas. Para observar las reacciones en el córtex (subcórtex), hay que eliminar el epicórtex o utilizar secciones transversales del talo. En Ozenda \& Clauzade (1970) y Clauzade \& Roux (1985), los autores interpretan el subcórtex como médula, mencionan una separación nítida entre esta y el estrato cortical, y le atribuyen las reacciones propias del subcórtex.

Debido a lo anteriormente expuesto existe una cierta confusión respecto a las especies de Roccella, que queda reflejada en la Checklist de la Península Ibérica (Hladun \& Llimona 2002-2007). El objetivo de este trabajo es aclarar y actualizar la nomenclatura de los taxones del género presentes en las costas peninsulares y Baleares, aportando una clave de identificación para los mismos y datos sobre su distribución.

\section{MATERIALES Y MÉTODOS}

El material estudiado, 230 muestras (Anexo I), procede de los herbarios BCN (que incluye el procedente de MUB y se reseña como Ex-MUB), MA, MACB, PO, SANT y Vigo.

De todas las muestras se prepararon manualmente secciones finas de las ramas para su estudio en microscopio óptico.
En aquellos ejemplares, en general poco numerosos, en los que aparecen ascomas, se realizaron preparaciones para estudiar los caracteres propios de esta estructura reproductora. También en todas las muestras se emplearon los reactivos usuales en el género, $\mathrm{C}$ y K en córtex/subcórtex, soralios y médula, en la que también se aplicó I/IK (reacción más rápida y nítida tras la aplicación de hipoclorito). Solamente se realizaron pruebas de TLC, según el método descrito por Orange et al. (2001), para confirmar Roccella elisabethae y en algunos ejemplares de $R$. maderensis y R. fuciformis. La utilidad de esta técnica es pequeña, pues casi todos los taxones estudiados contienen los mismos metabolitos secundarios y lo que varía entre ellos es la zona del talo en que se presentan.

\section{RESULTADOS}

Este apartado se inicia con una clave de identificación sencilla pero que consideramos eficaz para la mayoría de los ejemplares estudiados. A continuación se describen las especies ordenadas por orden alfabético, enumerando el basiónimo, el tipo y los sinónimos más utilizados; la descripción esta basada en los ejemplares estudiados. Se aportan datos sobre su distribución general y en la zona de estudio.

\section{Clave de identificación de las especies estudiadas}

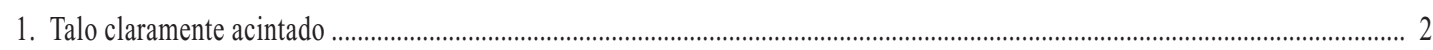

1. Talo de ramas cilíndricas o sólo ligeramente aplanadas...................................................................................................... 3

2. Córtex y soralios $\mathrm{C}+$ rojo-carmín ................................................................................................................. R. maderensis

2. Córtex C-. Soralios C+ rojo-carmín ................................................................................................................ R. fuciformis

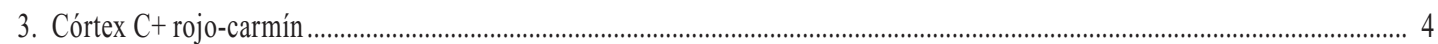

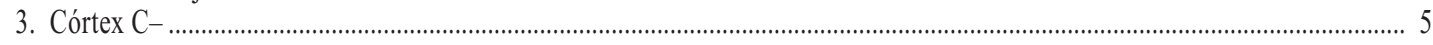

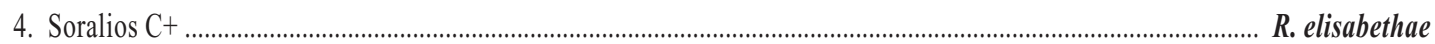

4. Soralios C-. Médula del disco de fijación amarilla ....................................................................................... R. phycopsis

5. Con apotecios o con soralios. Soralios $\mathrm{C}+$ rojo-carmín................................................................................................ R. tinctoria

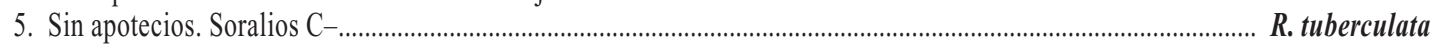

Roccella elisabethae Tehler, Symb. Bot. Ups. 34(1): 413(2004) En algunos ejemplares se observan pequeñas proliferacioTipo: Canary Islands, Tenerife, Buenavista del Norte, on the N-slope ca, con áreas negras en algunos ejemplares. Córtex de 70of Montaña de Talavera near La Cuesta, 28 21.435' N, $16^{\circ} 50.625^{\prime} \mathrm{W}, \quad 90 \mu \mathrm{m}$ de grosor, en plecténquima de hifas perpendiculares 300-350 m, 1-Jan-2001, Tehler 8306 (S, holotipo; B, BM, E, ESS, H, $\quad$ a la superficie de las ramas mezcladas con otras transver-
LD, NY, TFMC, UPS, US, isotipos).

Talo de $2-8 \mathrm{~cm}$ de longitud, de ramas cilíndricas, a veces algo aplanadas en la base y de color gris o pardo gris. sales, por lo que no se aprecia un tejido en empalizada perfecta. Más o menos en empalizada, según Aptroot \& Schumm (2011). Soralios siempre presentes, numerosos, 
de planos a convexos, circulares u ovoides. En algunos se presentan gruesos soredios y proliferaciones a modo de pequeñas ramas. Apotecios y picnidios desconocidos en la especie (Tehler et al. 2004).

Test químico. $\mathrm{C}+$ rojo carmín intenso y $\mathrm{K}$-, tanto en el córtex como en los soralios. Médula I/KI+ violeta casi negro. TLC: se detecta exclusivamente ácido lecanórico.

Observaciones. Roccella elisabethae se puede confundir con $R$. arnoldii Vain. En la descripción de R. elisabethae, Tehler estudia el tipo de Vainio y lo incluye en $R$. tinctoria, descartando la igualdad entre ambos taxones. Sin embargo, como advierten Aptroot \& Schumm (2011), en la mayoría de las claves y floras europeas (Ozenda \& Clauzade 1970, Poelt 1969, Clauzade\& Roux 1985), el concepto de $R$. arnoldii coincide con la especie de Tehler. Los ejemplares estudiados que estaban identificados como R. arnoldii corresponden a $R$. phycopsis.

Hábitat y distribución. Esta especie es siempre saxícola en rocas del litoral. Se conocía de Canarias, Madeira y Cabo Verde; por tanto, la cita de Aguiño en la provincia de A Coruña (Galicia), es la primera para la Península Ibérica y para Europa. Ya se conocían otras especies liquénicas macaronésicas que se presentan en el litoral gallego y constituyen también citas únicas para Europa (Paz-Bermúdez \& López de Silanes 1998, Paz-Bermúdez et al. 1998, Paz-Bermúdez 2000).

Roccella fuciformis (L.) DC., Fl. Franç. ed. 3(2): 335 (1805)

Lichen fuciformis L. Sp. Plant.: 1147 (1753).

Tipo: (icon in) Dillenius, Hist. musc. T.22, f. 61 A \& B (Jørgensen et al. 1994).

Talo de 4-12 cm de longitud, con ramas aplanadas de 2-10 mm de anchura. El color es muy variable, de gris con un tono malva a castaño oscuro. Superficie mate, con aspecto afieltrado. La médula del disco de fijación es de color negro. Córtex de 50-60 $\mu \mathrm{m}$ de grosor, en plecténquima en empalizada bien definido. Soralios siempre presentes, numerosos, en su mayoría situados en los bordes de las ramas; ovoides o circulares a veces confluentes; en general planos y con soredios finos. Los apotecios, poco frecuentes, rompen el córtex, por lo que en su inicio aparentan tener excípulo talino; variables en forma, de planos a convexos, sobre $1 \mathrm{~mm}$ de diámetro, con el disco negro a veces pruinoso. Himenio de aproximadamente $160 \mu \mathrm{m}$; epihimenio negro-verdoso; hipotecio grueso, de color castaño oscuro a negro. Ascos 90-120 × 12-16 $\mu \mathrm{m}$. Ascósporas incoloras con 3 septos, con ápices redondea- dos 0 apuntados, 24-32 × 4-6 $\mu \mathrm{m}$. Conidiomas y conidios no vistos en el material estudiado.

Test químico. Córtex C-. Soralios C+ rojo carmín, $\mathrm{K}+$ amarillento luego rojizo en el extremo de las ramas. Médula I/KI+ negro violáceo.

Hábitat y distribución. Todos los ejemplares estudiados son saxícolas, en rocas litorales o próximas a la costa. Se conoce de Macaronesia, el Mediterráneo occidental y en el Atlántico este desde Marruecos a Inglaterra. Se presenta en todo el litoral peninsular y en las islas Baleares, pero es mucho más frecuente en la costa atlántica.

Roccella maderensis (J. Steiner) Follmann, J. Hattori Bot. Lab. 32: 37 (1969), nom. invalidum; Courier Forschungsinst. Senckenberg 159: 180 (1993) nom. validum.

Tipo: Portugal, Madeira, Ponte Delgada (W-806 lectotypus)( Tehler et al. 2004: 416).

Roccella fuciformis (L.) DC. var. maderensis J. Steiner

Talo de longitud muy variable (4) $7-12 \mathrm{~cm}$; ramas en su mayoría acintadas, de 1-10 mm de anchura que en algunos ejemplares se mezclan con otras de sección ovoide. La médula del disco de fijación es negra o blanca y negra. Córtex de 40-60 $\mu \mathrm{m}$, en un claro plecténquima en empalizada. Médula de hifas intricadas. Son frecuentes los soralios, circulares u ovoides, a veces excavados, otras convexos, raramente confluentes. Se presentan también en los ejemplares con apotecios, que son raros pero mucho más comunes que en $R$. fuciformis. Apotecios, con excípulo talino perfectamente definido (diferencia con R. fuciformis), de 1-3 mm de diámetro, con disco negro casi siempre pruinoso. Himenio de 70-100 $\mu \mathrm{m}$, con epi- e hipotecio castaño-negruzco. Parafisoides ligeramente engrosados en el ápice. Ascos de 60-80 × 12-16 $\mu \mathrm{m}$. Ascósporas triseptadas, alguna curvadas, $24-30 \times 5-7 \mu \mathrm{m}$. Conidiomas en picnidios de aproximadamente $1 \mathrm{~mm}$, formando grupos visibles como manchas negras. Conidios arqueados de $12-16 \times 1-1,5 \mu \mathrm{m}$.

Test químico. Córtex, soralios y pruína de los apotecios $\mathrm{C}+$ rojo carmín. Extremos de las ramas y soralios $\mathrm{K}+$ amarillo que se vuelve rosado o rojizo. Médula $\mathrm{I} / \mathrm{KI}+$ negro violáceo.

Hábitat y distribución. Los ejemplares estudiados son todos saxícolas. Esta especie se conoce de Madeira, Azores y, en Europa, de dos localidades portuguesas: Cabo de Roca (Estremadura) e islas Berlengas (Beira Litoral).

Observaciones. Roccella allorgei des Abb., citada también de Estremadura (Portugal), se aproxima a R. maderensis por las reacciones con $\mathrm{C}$ y $\mathrm{K}$, pero difiere por la 
forma de las ramas, en su mayoría, cilíndricas. Según Tehler et al. (2004) y Tehler et al. (2009b), R. allorgei presenta tanto formas sorediadas, como fértiles, y más raramente individuos con los dos tipos de reproducción. Sus apotecios inmersos en el talo y sin excípulo talino son claramente diferentes a los de $R$. maderensis. Sin embargo, Aptroot \& Schumm (2011), ciñéndose a la descripción original de $R$. allorgei, consideran que los apotecios son desconocidos en la especie. Ninguna de las muestras estudiadas se puede incluir en este taxón.

Roccella phycopsis (Ach.) Ach., Arch. Syst. Naturgesch. 1(1): 110 (como Parmelia phycopsis) (1804). Lich. univ.: $440(1810)$

Lichen fucoides Dicks.

Tipo: s. loc., s.d., Dickson s.n., E neotipo (Tehler 2002).

Roccella pygmaea Mont. (1849).- Roccella fucoides Vain. (1901).

Talo fruticuloso, de 2-7 cm de longitud; algunos ejemplares forman almohadillas densas; ramas cilíndricas, de 0,5-1,5 mm de diámetro. En algunos individuos se forman abundantes ramas laterales cortas. El color es variable, de gris a castaño oscuro, en muchos talos el lado expuesto es mucho más oscuro que el interno. La superficie es lisa, mate, con aspecto afieltrado. Médula del disco de fijación, hipomédula, de color amarillo limón, carácter constante y propio de esta especie. Córtex de hifas perpendiculares a la superficie, formando un plecténquima en empalizada continuo, de 60-90 $\mu \mathrm{m}$ de grosor. Médula laxa de hifas desordenadas, menos densa en el centro. Soralios abundantes, al principio planos, circulares, bien delimitados, luego convexos, confluentes y tomando formas muy variadas. Los soredios frecuentemente son gruesos y en algunos se forman proliferaciones con aspecto de diminutos nuevos individuos. Apotecios muy poco frecuentes, convexos, de forma poco definida, sin excípulo talino, con el disco negro a veces ligeramente pruinoso, de $1 \mathrm{~mm}$ de diámetro y posición lateral en las ramas. En sección, no se observa excípulo talino ni propio. Himenio de 70$100 \mu \mathrm{m}$, con epitecio negro-verdoso e hipotecio castaño muy oscuro, casi negro. Parafisoides ligeramente ramificados en el ápice. Ascos claviformes 60-90 × 12-16 $\mu \mathrm{m}$. Ascósporas rectas, de 24-30 × 8-10 $\mu \mathrm{m}$, con tres septos y paredes gruesas. Conidiomas y conidios no vistos en el material estudiado.

Test químico. Córtex, C+ rojo-carmín, soralios $\mathrm{C}-$ (a veces ligeramente rosado), $\mathrm{K}+$ amarillo rojizo de intensidad variable. Médula I/KI+ azul intenso.
Hábitat y distribución. Frecuente en rocas del litoral, pero puede alejarse de la costa comportándose como saxícola o como epífita sobre muchos tipos de árboles, arbustos y matas. Se presenta en todo el Mediterráneo, en Macaronesia y en el Atlántico este desde Marruecos hasta el norte de Inglaterra. En la Península, es la especie más frecuente, tanto en la costa atlántica como en la mediterránea donde se comporta muchas veces como epífita.

\section{Roccella tinctoria DC., Fl. Franç. ed. 3(2): 334 (1805)}

\section{Lichen roccella L. Sp.P1.:1154 (1753).}

Tipo: Roccella ex insula Fyal, s.ann., s.coll., s.n. LINN.1273.263 (lower specimen) lectotipo (designado por Howe (1912) y reasignado por Jørgensen et al. 1994).

Roccella arnoldii Vain. (1901).- Roccella boergesenii Vain. (1924).Roccella canariensis Darb. (1898).- Roccella guanchica Eige \& Viethen (1987)?.- Roccella tuberculata Vain. var. vicentina Vain.(1901).Roccella vicentina (Vain.) Follmann (1987).

Talo de 3-15 cm de longitud, con ramas cilíndricas (0 ligeramente aplanadas) de color pardo oscuro en el lado expuesto y gris amarillento en el protegido. La superficie mate, lisa en las ramas superiores, rugosa o con fisuras anulares hacia la base del talo. La médula del disco de fijación es blanca con áreas negras. En sección se observa un epicórtex de color pardo más o menos intenso, delgado, 3-4 um; el subcórtex, de hifas entrelazadas, hialino, también delgado, 15-35 um de grosor. Médula de hifas gruesas que se introduce en algunos puntos en la capa algal. Soralios abundantes, muy variables de forma y tamaño, a veces confluentes; con soredios muy gruesos; en algunos ejemplares, en los soralios se forman pequeñas ramas que aparentan el inicio de nuevos individuos. Apotecios, muy raros en las muestras de la Península, de 1-2 mm de diámetro, con excípulo talino patente, generalmente planos con disco negro, casi siempre pruinoso. Himenio 120-140 $\mu \mathrm{m}$, con hipotecio y epitecio castañonegruzco. Ascos de 60-80 × 14-18 $\mu \mathrm{m}$. Ascósporas, hialinas, triseptadas, ligeramente curvadas, con ápices obtusos, $20-25 \times 4-6 \mu \mathrm{m}$. Picnidios no vistos en el material estudiado.

Test químico. Córtex C-. Subcórtex y pruina de los apotecios $\mathrm{C}+$ rojo carmín, $\mathrm{K}-$. Médula I/KI+ negro violáceo.

Observaciones. R. canariensis/R. tinctoria son consideradas «especies par», la primera con reproducción sexual, la segunda sólo con reproducción vegetativa mediante soralios, que actualmente han sido unificadas bajo el 
nombre de $R$. tinctoria DC. (Theler et al. 2009a). Algunas floras europeas (Ozenda \& Clauzade 1970, Clauzade \& Roux 1985) atribuyen a $R$. canariensis una reacción C+ en el córtex, un error con pocos efectos prácticos dada la rareza de ejemplares con apotecios en Europa (en el material estudiado sólo presentes en los ejemplares de Cabo de Roca e Islas Berlengas).

Hábitat y distribución. Todos los ejemplares estudiados son saxícolas, en rocas costeras. Se encuentra en las costas del Atlántico este, desde Angola hasta el norte de Galicia, en la Macaronesia y en algunas áreas del Mediterráneo occidental. En la Península es mucho más común en el litoral atlántico que en el mediterráneo.

Roccella tuberculata Vain., Catal. Welwitsch Afric. Plants, 2: 433 (1901)

Tipo: Cabo Verde. «Ad rupes in insula Fogo», leg. Pharmacop. Torres. No. 419. Ster» BM 660995 lectotipo (Tehler et al. 2004).

Roccella tuberculata Vain. var. tuberculata Vain

Talo de $1-5 \mathrm{~cm}$ de longitud, de ramas cilíndricas, ligeramente aplanadas en la base, rígido, con la superficie de mate a ligeramente brillante, no afieltrada, de color castaño oscuro en la parte expuesta y pardo claro en la protegida. La médula del disco de fijación varia de blanca a amarillo ocre, no amarillo limón como en Roccella phycopsis. La sección de las ramas muestra un epicórtex amarillento o hialino, de 3-4 $\mu$ m y un subcórtex, de 30$60 \mu \mathrm{m}$, formado por hifas entrelazadas, no en empalizada. Capa algal mal delimitada. Médula laxa. Soralios abundantes, en su mayoría circulares, planos, bien delimitados por un reborde del talo, pero a veces convexos y poco definidos. Apotecios desconocidos en la especie. Tampoco se conoce especie alguna que los presente y forme par con ella. Picnidios no vistos.

Test químico. Córtex y soralios C- (en los extremos de las ramas puede dar un ligero rojo debido al escaso desarrollo del epicórtex). Subcórtex, $\mathrm{C}+$ rojo carmín, $\mathrm{K}+$ rojizo en el extremo de las ramas. Médula I/IK+ azul.

Hábitat y distribución. Siempre saxícola. Se conoce de la región Macaronésica, Cabo Verde y de la costa atlántica de España y Portugal. Egea (1989) recoge alguna cita dudosa del Mediterráneo. Es una especie poco frecuente en el litoral ibérico.

\section{CONCLUSIONES}

El género Roccella ha experimentado en los últimos años cambios notables al sinonimizarse varias especies y unificarse las consideradas como «especies par». Por ello, las 10 especies mencionadas en la Checklist de la Península Ibérica y Baleares se reducen a 7: 6 estudiadas en este trabajo; la séptima, $R$. allorgei, está citada de Cabo de Roca, Sintra (Portugal), como única cita en Europa. R. maderensis (Sintra e Islas Berlengas) y R. elisabethae (Aguiño) constituyen también citas únicas para nuestro continente. $R$. tuberculata se presenta en el litoral atlántico y es una especie poco frecuente. $R$. fuciformis, $R$. tinctoria y $R$. phycopsis se encuentran tanto en la costa atlántica como mediterránea, siendo la última la más común en el litoral peninsular.

\section{AGRADECIMIENTOS}

Este trabajo fue financiado por los proyectos DGI CGL200404795-C04-03/ BOS y DGI CGL2007-66734-C03-03/ BOS del Ministerio de Educación e Innovación.

\section{BIBLIOGRAFÍA}

Aptroot, A \& Schumm, F. 2011. Fruticose Roccellaceae an anatomical-microscopical atlas and guide with a worldwide key and further notes on some crustose Roccellaceae or similar lichens. F. Schumm, Wangen.

Clauzade, G. \& Roux, C. 1985. Likenoj de okcidenta Eúropo. Bull. Soc. Bot. Centre-Ouest Nouvelle Série n. special, 7: $1-893$.

EGEA, J. M. 1989. Las comunidades liquénicas saxícolas, ombrófobas, litorales del Suroeste de Europa y Norte de África (Roccelletea phycopsis classis prov.). Studia Geobotanica 9: 73-152.

Follmann, G. 1969. En: J. Poelt (Ed.), Bestimmungsschlüssel europäischer Flechten: 704-706. Cramer, Lehre.
Hladun, N. \& Llimona, X. 2002-2007. Checklist of the lichens and lichenicolous fungi of the Iberian Peninsula and Balearic Islands. http://botanica.bio.ub.es/checklist/check-list.htm.

Orange, A.; James, P. W. \& White, F. J. 2001. Microchemical methods for the identification of lichens. British Lichen Soc., London.

Ozenda, P. \& Clauzade, G. 1970. Les Lichens. Masson \& Cie., Paris.

Paz-Bermúdez, G. 2000. Lecanographa dialeuca, new to Europe. Lichenologist 32: 405-407.

Paz-Bermúdez, G.; Carballal, R. \& López de Silanes, M. E. 1998. Two new records of interesting species to the European flora. Lichenologist 30: 591-593. 
Paz-Bermúdez, G. \& López de Silanes, M. E. 1998. Bactrospora carneopallida, new to Europe. Lichenologist 30: 291-293.

PoELT, J. 1969. Bestimmungsschlüssel europäischer Flechten. Cramer, Lehre.

TAVARES, C. N. 1958. On the taxonomy of some Roccella species of the «canariensis» group. Rev. Fac. Cienc. Lisboa 6(2): 125-144.

Tehler, A. \& Irestedt, M. 2007. Parallel evolution of lichen growth forms in the family Roccellaceae (Arthoniales, Euascomycetes). Cladistics 23: 432-454.

Tehler, A.; Dahlkild, Å; Eldenäs, P. \& Feige, G. B. 2004. The phylogeny and taxonomy of Macaronesian, European and Mediterranean Roccella (Roccellaceae, Arthoniales). Symbolae Bot. Upsalienses 34(1): 405-428.

Tehler, A.; Irestedt, M.; Wedin, M. \& ErTz, D. 2009a. Origin, evolution and taxonomy of American Roccella (Roccellaceae, Ascomycetes). Systematics and Biodiversity 7(3): 307-317.

Tehler, A.; Irestedt, M.; Bungart, F. \& Wedin, M. $2009 \mathrm{~b}$. Evolution and reproduction modes in the Roccella galapagoensis aggregate (Roccellaceae, Arthoniales). Taxon 58: 438-456.

Anexo I. Material estudiado

\section{Roccella elisabethae Tehler}

ESPAÑA: CoRuÑa, A: Aguiño, 15-8-1971, M. Horjales, MA-Lichen 4572; Aguiño, 15-8-1971, M. Horjales, MACB 8585 (Identificadas como Roccella vicentina). Otro material estudiado: ESPAÑA: Islas Canarias: Lanzarote, Haría, Peñas del Chache, aprox. $500 \mathrm{~m}$, 13-1-1990, J. M. Egea, Ex-MUB 50078; Lanzarote, Orzola, Peñas de Andia, aprox. 50 m, 13-1-1990, J. M. Egea, Ex-MUB 50095, 50103. Tenerife, Guimar, mirador, aprox. 400 m, 11-1-1990, J. M. Egea, EXMUB 50111, 50114 (identificadas como Roccella sp.).

\section{Roccella fuciformis (L.) DC}

ESPAÑA: Almería: Cabo de Gata, Playa del Monsul, 10-41995, R. Novoa, SANT- Lich. 11703. CádIz: Zahara de los Atunes, Sierra del Retín, 30STF45408, 300m, sobre areniscas ácidas en paredes verticales orientación NW, 13-8-1983, J. García-Rowe, MACB 39119, SANT-Lich 8444, Ex-MUB 50021. ConuÑa, A: Islas Sisargas, 6-1978, L. Bahillo, SANT-Lich 1143; Finisterre, 26-2-1978, L. Bahillo, SANT-Lich 1144; A Capelada, Punta Candelaria, 29TNJ7640, 150 m, sobre peridotitas, 14-2-1992, M. J. Sánchez-Biezma, G. Paz \&M. E. López de Silanes, SANT-Lich 9232; Cabo Laxe, 10-9-1997, G. Paz-Bermúdez, SANT-Lich 9940; Muxía, Santuario de Nosa Señora da Barca, 12-05-2003, R. Carballal \& G. Paz-Bermudez, SANTLich 11552; Santa Eugenia de Ribeira, Aguiño, rocas marítimas, 15 8-1971, M. Pastrana, MACB 5272, 5273; Corrubedo, rocas cerca de la laguna de Monte Vixán, 10-11-1991, Alberto, Vigo-Lich. 443. MÁLAGA: Playas de Manilva, 100 m., 5-1989, J. M. Egea, Ex-MUB 50023. Murcia: Cabo de Palos, Carmolí, rocas volcánicas, 11-21978, Esteve, Ex-MUB 1551; Cabo de Palos, Isla Mayor, rocas volcánicas, 25-3-1975, J. M. Egea, Ex-MUB 1552. Pontevedra: Isla de La Toja, 15-11-1953, sin otros datos, SANT-Lich 320; Isla de Ons, 100 m, 7-1988, J. M. Egea, Ex-MUB 50032; 0 Grove, Punta Abelleira, 29TNH0600, en roca granodiorítica, 23-3-1996, A. García \& M. Freire, Vigo-Lich 1317, 1318; 0 Grove, Playa Mexilloeira, 29TNH0803, roca granodiorítica, 14-5-1996, A. García \& M. Freire, Vigo-Lich. 1319; O Grove, Punta Pateiro, 29TNH0502, roca granodiorítica, 14-5-1996, A. García \& M. Freire, Vigo-Lich. 1320; 0 Grove, A Airadella, 29TNH0902, roca granítica, 159 m, 12-10-1995, $A$. Garcia \& M. Freire, Vigo-Lich. 1325. IsLAs BaLEARES: Formentera, Torrent de Ses Fontanelles, 1979, L. Fiol, BCN 2702.

PORTUGAL: BEIRA LiTORAL: Islas Berlengas, sin fecha, Welwitsch, PO 5387 L; Islas Berlengas, 7-1989, J. M. Egea, Ex-MUB 50016, 50017, 50057, 50059, 50065, 50066, 50067 (todos los ejem- plares como Roccella sp.). Douro LitoraL: Serra do Pilar, estrada marginal, 1-1880, I. Newton, PO 5386 L; Valongo, Roboredo, 18-71915, G. Sampaio, P0 948 L. Estremadura: Sintra, Cabo de Roca, 19-2-1987, J. M. Egea, Ex-MUB 50010; Sintra, Cabo de Roca, 4-71989, J. M. Egea, Ex-MUB 50075 (como Roccella sp.).

Otro material estudiado: ESPAÑA: IsLas CANARIAS: Tenerife, Puerto de la Cruz, Taoro, 120 m, en basalto, 18-6-1957, O. Klement, MA-LICH 307; Tenerife, mirador de Güímar, 400 m, 13-1-1984, J. M. Egea \& X. Llimona, Ex-MUB 9103; Gomera, carretera de AguloVallehermoso, km 37, 335 m, roquedo, 8-8-1994, J. Etayo, MA-LICH 6510. Gran Canaria, Agaete, Anden Verde, 3-1-1984, Naranjo \& J. M. Egea, Ex-MUB 9104; Lanzarote, Orzola, Peñas de Andia, 50 m, 13-1-1990, J. M. Egea, Ex-MUB 50104.

\section{Roccella maderensis (J. Steiner) Follmann}

PORTUGAL: BeIRA LitoRAL: Islas Berlengas, 7-1989, J. M. Egea, Ex-MUB 50014, Ex-MUB 50018, 50042, 50052, 50122 (identificadas como Roccella sp.); Insula Berlenga, prope castrum, ad rupes graniticas, 20 m, 14-4-1951, C. N. Tavares, PO 5060 L Lichenes Lusitaniae selecti exsiccati, $n^{0} 30$, (identificada como Roccella fuciformis Ach.). Estremadura: Sintra, Cabo de Roca, 20-140 m, 19-21987, J. M. Egea, Ex-MUB 50022, 50024, 50025; Sintra, Cabo de Roca, 4-7-1989, J. M. Egea, Ex-MUB 50044, 50045, 50050 (las tres últimas identificadas como Roccella sp.). Otro material estudiado: PORTUGAL: MadeIRa: San Vicente, $130 \mathrm{~m}$, en comunidades de Sonchetum pinnati (Visneamocanero-Apollonion barbujanae Rivas Martínez in Capelo et al. 2000), 9-9-2004, E. Fuertes, MA-Lichen 15219; Madeira, San Vicente, $130 \mathrm{~m}$, en fisuras de rocas basálticas (casmófita), en comunidades de Sonchetum pinnati, 9-9-2004, E. Fuertes, MALichen 15785, MACB 89975 (todos identificados como Roccella fuciformis Ach.)

\section{Roccella phycopsis (Ach.) Ach.}

Selección del material estudiado sobre 95 muestras. ESPAÑA: Alicante: Jávea, Cabo San Martín, BC 5994, sobre Pinus halepensis, 2-5-1986, J. M. Egea \& P. Torrente, Ex-MUB 50030; Calpe, Peñón de Ifach, BC 4580, 332 m, 19-6-1987, J. M. Egea, P. P. Moreno \& F. L. Alonso, Ex-MUB 21290. Almería: Almería, proximidades del Puente del Cura, WF7643, 80 m, sobre calizas, 18-3-1990, J. M. Egea \& F. L. Alonso, Ex-MUB 21293. Castellón de la Plana: Isla Columbrete Mayor, El Mascarat, 31SCE0218, $20 \mathrm{~m}$, sobre rocas volcánicas, 10-7-1985, F. Boisset, Ex-MUB 50026. CoRUÑa, A: Or- 
tigueira, A Capelada, 29TNJ9146, 348 m, sobre eclogitas, 6-3-1992, Sánchez-Biezma, M. J., Paz, G. \& López de Silanes, M. E., SANTLich 9233; Muxía, Santuario Virxen da Barca, sobre granito, 12-52003, Carballal, R. \& Paz-Bermúdez, G., SANT-Lich 11557. GiroNA: Tossa de Mar, La Selva, 31TDG92, sobre Quercus suber, 10-91976, X. Llimona, BCN 3949; Alt Empordá, Illes Medes, EG 15, sobre Olea europaea, 4-3-1972, A. Gómez-Bolea, BCN 3280. GRANADA: Almuñécar, La Herradura, Cerro Gordo, VF 6530, 315 m, 253-1989, J. M. Egea, Baeza \& F. L. Alonso, Ex-MUB 21288. MálaGa: Manilva, Punta de la Chullera, 5-30 m, 5-1989, J. M. Egea, Ex-MUB 50040. Murcia: Mazarrón, Cabo Tiñoso, La Picadera, sobre Rhamnus, 21-3-1987, J. M. Egea, P. P. Moreno \& F. L. Alonso, ExMUB 50028; Cabo de Palos, Cabezo de la Fuente, YG0368, 10 m, sobre calizas y dolomías, 22-12-1989, J. M. Egea \& F. L. Alonso, Ex-MUB 21289. Pontevedra: 0 Grove, Punta Abelleira, 29 TNH 0600, en roca granítica supralitoral, 23-03-1996, A. García \& M. Freire, VIGO-Lich. 1322; Isla de Ons, 7- 1988, J. M. Egea, Ex-MUB 50000. Islas BaLEares: Eivissa, Las Puertas del Cielo, sobre Rosmarinus officinalis, 9-09-2008, R. Arroyo, E. Seriñá \& E. Araujo, MACB 98925. Formentera, Es Ram, sobre Juniperus, 10-09-2008, M. E. López de Silanes \& G. Paz Bermúdez, SANT-Lich 11382. Mallorca, Porto Cristo, cala cerca de las cuevas del Drac, sobre calizas, 147-1994, J. Etayo, MA-Lichen 6731.

PORTUGAL: Algarve: Lagos, Ponta da Piedade, en rocas 100 m, 16-2-1987, J. M. Egea, Ex-MUB 50038. Beira Litoral: Leiria, nas murallas no castelo, 6-9-1918, A. Ricardo Jorge, P0 6638 L.; Islas Berlengas, 7-1989, J. M. Egea, EX-MUB 50042 (como Roccella sp.). Estremadura: Ericeira, en pedras dun muro antigo, orientación Norte, 30 m, 2-2-1964, C. N. Tavares, PO 5190 L; Sintra, cabo de Roca, 4-07-1989, J. M. Egea, Ex-MUB 50119.

Otro material estudiado: ESPAÑA: IsLas CanaRIas: Gran Canaria, Las Palmas, Isleta, 8-1-1984, J. M. Egea, Ex-MUB 9111. FRANCIA: Brignogan, Finistère, 1897, Herbier A. Boistel, MA-Lichen 2706. GRECIA: Ins. Mikonos, 6-1927, K. H. Rechinger, MA-Lichen 434, Cryptogamae exsiccatae editae Museo Hist. Natur. Vindobonensi $n^{0}$ 1027. ITALIA: Sicilia: Siracusa, entrada a Latomie del Paradiso, 50 m, pared caliza vertical, 28-4-1961, M. Steiner, MA-Lichen 173, Cryptogamae exsiccatae editae Museo Hist. Natur. Vindobonensi ${ }^{0}$ 4336. MARRUECOS: Sidi Ifni, cerca de la ciudad, 10-4-1987, M. E. López de Silanes, SANT-Lich 11689; Agadir, Tanasahor, Cabo Rhir, 84-1987, M. E. López de Silanes, SANT-Lich 11691. TURQUÍA: Trabzon: Koprabasi, Costa del Mar Negro, entre Arakli y Arsin 40 57' N $39^{\circ}$ 59'E., 20 m, acantilados silíceos, 30-7-1997, V. John, BCN 16937 , V. John: Lichenes Anatolici Exsiccati n ${ }^{0} 112$.

\section{Roccella tinctoria DC.}

ESPAÑA: Almería: Sierra de Gata, Boca de los Frailes, $12-$ 1972, X. Llimona, BCN-Lich 1148; Cabo de Gata, cerro San Miguel, rocas, 21-3-1972, E. Fuertes \& C. Morales, MACB 432078 (como R. tuberculata var. vicentina); Cabo de Gata, subida al collado de Vela Blanca, andesitas, 13-9-1982, J. M. Egea, Ex-MUB 1259; Cabo de Gata, Vela Blanca, 200 m, 6-1987, J. M. Egea, Ex-MUB 50004. CoruÑA, A: Corrubedo, 20-50 m, 7-1988, J. M. Egea, Ex-MUB 50009; Finisterre, 26-2-1978, L. Bahillo, SANT-Lich 8137; Islas Sisargas, 6-1978, L. Bahillo, SANT-Lich 8147; A Capelada, Ortigueira, piedras de acantilado próximas al faro de Cabo Ortegal, 29TNJ9146, $200 \mathrm{~m}$, sobre gneis-eclogita, 6-3-1992, M. J. Sánchez-Biezma, G. Paz \& M. E. López de Silanes, SANT-Lich 9234; A Capelada, Ortigueira, saliente rocoso próximo a Pico Gargacido, 29TNJ9046, $351 \mathrm{~m}$, sobre gneiseclogita, 6-3-1992, M. J. Sánchez-Biezma, G. Paz \& M. E. López de
Silanes, SANT-Lich 9235; A Capelada, Cedeira, saliente próximo a Punta Candelaria, 29TNJ7640, 150 m, sobre peridotita, 14-2-1992, M. J. Sánchez-Biezma, G. Paz \& M. E. López de Silanes, SANT-Lich 9236; Muxía, Santuario Nosa Señora da Barca, 12-5-2003, R. Carballal \& G. Paz-Bermudez, SANT-Lich 11692. Pontevedra: Cangas de Morrazo, Punta Alada, 6-7 m, 4-10-1986, M. E. López de Silanes, SANT-Lich 11687; 0 Grove, Punta Abelleira, 29TNH0600, roca granítica, adlitoral, 23-3-1996, A. García \& M. Freire, Vigo-Lich. 571 (como R. boergesenii Vainio); 0 Grove, punta Abelleira, 29TNH0600, roca granítica, supralitoral, 23-3-1996, A. García \& M. Freire, VigoLich. 1326, Vigo-Lich. 1327; Illas Cíes, 29TNG0773, cantil na illa do medio, granito de dos micas, 175 m, 19-4-1997, G. Paz-Bermúdez, SANT-Lich 9694; Oia, cerca de Mariñas, 29TNG0860, rocas muy expuestas, parte alta del supralitoral, granito, 24-5-1998, F. Méndez \& A. García, Vigo-Lich. 911. Todos los ejemplares, identificados como Roccella vicentina Vain., excepto dos cuya identificación se especificó en el listado.

PORTUGAL: BeIRA Litoral: Islas Berlengas, 7-1989, J. M. Egea, Ex-MUB 50123 (como Roccella sp.). Estremadura: Sintra, Praia da Ursa, prope Cabo da Roca, ad rupes graniticas abruptas, occidentem spectantes, 100 m, 17-4-1953, C. N. Tavares, P0 5072 L, Lichenes Lusitaniae selecti exsiccati $\mathrm{n}^{0} 54$ como Roccella tuberculata Vain. var. vicentina Vain.; Sintra, Cabo Roca, 20-140 m, 19-2-1987, J. M. Egea, Ex-MUB 50003, 50007, 50008, 50011, 50012 (todas como R. vicentina); Sintra, Cabo Roca, 20-140 m, 19-2-1987, J. M. Egea, Ex-MUB 50019, 50020 (como R. canariensis); Sintra, Cabo Roca, 47-1989, J. M. Egea, Ex-MUB 50015 (ejemplar con apotecios), Ex-MUB 50043, 50053, 50119, 50060, 50072 (como Roccella sp.).

Selección de otro material estudiado: ARGELIA: Iles Habibas, rocas riolíticas cerca del faro, $100 \mathrm{~m}, 3-5-1934$, Dr. R. Maire, MALICH. 3118, Exsicc. Ch. Dufour n ${ }^{0} 7656$, como R. phycopsis. CAB0 VERDE: San Vicente, flanco suroeste de Monte Verde por encima de Mindelo, andesitas, en Roccelletum canariensis Follm., 450 m, 4-1974, G. Follmann, BCN-Lich 1710, Lichenes Exsiccati Selecti Museo Hist. Nat. Casselensi n ${ }^{0} 220$, como R. canariensis. ESPAÑA: IsLas CANARIAS: Gran Canaria, Cuesta de Silva, 100 m, 3-1-1984, Naranjo \& J. M. Egea, Ex-MUB 9101; Gomera, Valle del Gran Rey, afloramientos en el cruce con Las Sabinas, 1240 m, 8-8-1994, J. Etayo, MA-LICH 6508; Hierro, 200 m, 7-1981, C. Hernández \& P. L. Pérez, MACB 8877; La Palma, El Time, ad saxa vulcanica, 2-1980, G. B. Feige, BCN-Lich 5314, A. V?zda: Lichenes Selecti Exsiccati n² 2444, como R. tuberculata, quimiotipo vicentina; Tenerife, prope Los Cristianos, in littore, ad saxa maritima, 2-1984, G. B. Feige, BCN-Lich 5317, A. V?zda, Lichenes Selecti Exsiccati n ${ }^{0} 2442$, como R. canariensis Darb. MARRUECOS: Sidi-Ifni, a $5 \mathrm{~km}$ de la ciudad, 10-4-1987, E. López de Silanes, SANT-Lich 11689, 11690, ejemplar con apotecios. PORTUGAL: MADEIRA: Insula Bugio, ad rupes prope mare, 1945, J. G. Barreto, P0 5108 L. C. N. Tavares: Lichenes Lusitaniae selecti exsiccati $n^{0} 103$, como R. canariensis.

\section{Roccella tuberculata Vain.}

ESPAÑA: CoRuÑa, A: Santa Eugenia de Ribeira, Aguiño, rocas marítimas, 15-8-1971, R. Carballal, SANT-Lich 8145; Aguiño, 15-81971, M. Horjales, MA-Lichen 4572, MACB 8585; Islas Sisargas, 61978, L. Bahillo, SANT- Lich 8146; Finisterre, 26-2-1978, L.Bahillo, SANT-Lich 8136; Corrubedo, 20-50 m, 7-1988, J. M. Egea, Ex-MUB 50005, 50090; Cabo Laxe, 10-09-1997, G. Paz-Bermúdez, SANT-Lich 9941. Pontevedra: O Grove, Punta Abelleira, 29TNH0600, roca granodiorítica, supralitoral, 23-3-1996, A. García \& M. Freire, Vigo-Lich. 511; O Grove, Punta Pateiro, 29TNH0502, roca granítica, adlitoral, 14- 
5-1996, A. García \& M. Freire, Vigo-Lich. 1324; Oia, cerca de Mariñas, 29TNG0860, roca granítica, pared vertical, parte alta del supralitoral, 24-5-1998, F. Méndez \& A. García, Vigo-Lich. 910.

PORTUGAL: BEIRA LitoRAL: Leiria, nas muralhas do castelo, sin fecha, A. Ricardo Cunha, PO $6639 \mathrm{~L}$.

Otro material estudiado: ESPAÑA: IsLas CANARIaS: Gran Cana- ria, Custa de Silva, 100 m, 3-1-1984, Naranjo \& J. M. Egea, Ex-MUB 9118. Lanzarote, sin fecha, C. Hernández-Pacheco, MA-Lichen 2700. Tenerife, El Médano, 0-171 m, 10-1-1984, J. M. Egea, X. Llimona \& C. Hernández-Padrón, Ex-MUB 9093. Tenerife, Buenavista del Norte, Casa Blanca, 300 m, 10-1-1990, J. M. Egea, Ex-MUB 50083. 